\title{
The role of ultrasound and intravenous urography in evaluating patients with hematuria
}

\begin{tabular}{c}
\hline Ahmad Noah Hamudi Salih* \\
Abstract
\end{tabular}

Background and objective: Intravenous urography is a radiographic examination in which anatomic and physiologic abnormalities of the urinary tract are detected by obtaining a timed series of imaging of the abdomen and pelvis after the injection of intravenous "IV" iodinated contrast media. This study aimed to measure the diagnostic validity of ultrasound and intravenous urography in the hope of omitting unnecessary intravenous urography and to determine whether ultrasound could help to replace the intravenous urography in the diagnosis of hematuria.

Patients and methods: A prospective hospital based study was performed from $15^{\text {th }}$ April 2015 to $15^{\text {th }}$ April 2016 on 100 patients presenting to Rizgary Teaching Hospital with hematuria, all patients underwent a real time ultrasound examination of the urinary tract followed by an intravenous urography. The diagnostic validities were recorded and compared for each modality.

Results: Ultrasound had higher sensitivity than intravenous urography for diagnosis of kidney calculi, lower ureteric calculi, and urologic neoplasms, but in calculi of the middle and upper ureter, there was no difference between ultrasound and intravenous urography.

Conclusion: Our results are in favor of using ultrasound in the initial evaluation of hematuria. However, we must choose our diagnostic tool according to the patient's condition and suspected disorders causing hematuria, as ultrasound can be safely done and hence minimizing the exposure of the patient and medical staff to excessive radiation.

Keywords: Hematuria; Ultrasonography; Intravenous urography; Urologic neoplasm; Urinary calculi.

\section{Introduction}

The kidneys are bilaterally paired reddish brown organs. Typically each kidney weighs about $150 \mathrm{gm}$ in the male and $135 \mathrm{gm}$ in the female. The kidneys generally measure 10 to $12 \mathrm{~cm}$ vertically, 5 to $7 \mathrm{~cm}$ transversely, and $3 \mathrm{~cm}$ in the anteroposterior dimension. ${ }^{1}$ In healthy young adults, parenchymal thickness is of the order of $2.5-3 \mathrm{~cm}$ at the poles and $1.5-2 \mathrm{~cm}$ elsewhere. ${ }^{2}$ The ureters are bilateral tubular structures responsible for transporting urine from the renal pelvis to the bladder. They are generally $22-30 \mathrm{~cm}$ in length with a wall composed of multiple layers. ${ }^{1,4}$ The urinary bladder is a pyramidal muscular organ when empty. It has a triangular shaped base posteriorly. ${ }^{3}$ Hematuria is defined as the presence of red blood cells in the urine.When visible to the patient it is termed gross hemturia. Microscopic hematuria is that detected by the dipstick or microscopic examination of the urinary sediment. The more common cause of hematuria includes urinary tract infection, urolithiasis, trauma, renal parenchymal disease, and malignancy. Urinary tract malignancy is four times more common in patient with macroscopic hematuria than microscopic hematuria. ${ }^{5,6}$ The KUB radiograph is of limited value in the assessment of hematuria.However, it does play a role in the diagnosis of urinary stones, which may cause hematuria. ${ }^{7}$

* Department of Radiology, Rizgary Teaching Hospital, Erbil, Iraq.

** Department of Surgery, College of Medicine, Hawler Medical University, Erbil, Iraq. 
Ultrasound plays a central role in the assessment of hematuria. It is a readily available, risk-free and inexpensive imaging technique. It is a useful modality for the detection of urinary tract calculi. In addition, ultrasound has been shown to be more sensitive than intravenous urography (IVU) in diagnosing urological malignancy. The mass could be detected by ultrasound if it is more than $0.5 \mathrm{~cm} .{ }^{8}$ IVU was one of the first contrast based radiological investigations. It can provide information about the existence, etiology, location, and severity of obstruction. IVU may fail to detect radiolucent stone, small stone size (less than $5 \mathrm{~mm}$ ), or poor opacification of the renal collecting system due to severe obstruction. $^{9-11}$ Another disadvantage is that IVU may be protracted in patients with obstruction, when delayed images may be required several hours after injection of contrast for complete assessment. ${ }^{12}$ IVU involve the use of ionizing radiation and an intravenous contrast medium (CM). ${ }^{13,14}$ Non-ionic and low osmolar contrast medium, e.g., iohexol (ominipaque) have been shown to cause fewer serious side effects than ionic medium, e.g., diatrizoate (urographin). The quantity of CM administered to the patient by IV injection should be related to the weight of the patient $\left(300 \mathrm{mg} / \mathrm{kg}\right.$ body weight). ${ }^{15}$ Although IVU is still the in the nonglomerular hematuria, some clinicians use ultrasound in practice. We performed this study to compare the diagnostic value of ultrasound and IVU as the initial evaluation method in patients with hematuria. This study aimed to evaluate the feasibility of replacing IVU with ultrasound as the first line diagnostic modality for cases of patient with hematuria in our hospitals. The main objectives were to measure the diagnostic validity of ultrasound and IVU in the hope of omitting unnecessary and time consuming IVU and to determine whether ultrasound could help to replace the IVU in the diagnosis of patients with hematuria. ultrasound could help to replace the IVU in the diagnosis of patients with hematuria.

\section{Methods}

Patient Selection: This is a prospective hospital based study included a convenience sample of hundred patients with hematuria, who were referred for IVU in the radiology department of Rizgary Teaching Hospital; all patients underwent a urinary tract ultrasound and IVU examination. Patients with impaired renal function and history of allergy to iodinated contrast media were excluded.

Methods: All patients underwent a preliminary ultrasound examination of the urinary tract using an ultrasound machine $3.5 \mathrm{MHz}$ convex array transducer manufactured by Philips; Origin: Bothell, WA USA; Model No. HDXE version 2010. All patients were thoroughly examined in multiple planes (longitudinal, transverse and oblique axis) in both supine left and right lateral position. The urinary bladder was examined when adequately full. A report was provided at the completion of ultrasound examination. After the ultrasound the patient underwent an IVU examination using an $\mathrm{x}$-ray machine with the fluoroscope (iconos) with control, $500 \mathrm{mAs}$, manufactured by Siemens; origin Germany; Model 2005, the choice of film sequence was determined by the radiologist supervising the procedure. The contrast media used was water soluble iodinated low osmolar non-ionic contrast. $1 \mathrm{ml} / \mathrm{kg}$ body weight of iohexol (ominipaque 350) was manually injected intravenously via a median antecubital vein cannula.

\section{Image Analysis}

The analysis of IVU: All IVU images were analyzed for renal size, shape, and position, pelvicalyceal system and ureters for any features of dilatation or obstructive hydronephrosis(distension and dilation of the renal pelvis and calyces) or Hydroureter (dilatation of the ureter more than $3 \mathrm{~mm}$ ), filling defects or compression by space occupying lesion and for detection of urinary calculi. 
Ultrasound results analysis: the Urinary system was evaluated for urinary calculi, obstructive changes or other urinary tract causes of renal pain (e.g., space occupying lesions) or nonurinary tract alternate findings (e.g., pathologies related to the surrounding organs and structures like acute appendicitis, cholecystitis, and ovarian cyst). What is relevant for our study was the detection of urinary calculi, urinary system masses, their sites and presence of associated obstructive changes and the degree of obstruction.

Ethical considerations: Regarding ethical consideration, informed consent to participate in the study was obtained from all patients to obtain their agreement. The nature and the aim of the study explained to all participants through a direct interview, and verbal consent was taken from them beforehand.

Statistical analysis: The statistical analyses were carried out with the statistical package for the social sciences (version 19.0 for Windows). Descriptive analysis, including mean and standard deviation for quantitative variables and frequency, were performed, McNemar test was used and a $P$ value less than 0.05 was considered statistically significant. Each entry was double checked to avoid any possible mistakes.

\section{Results}

A total of 100 participants were enrolled in the study with the mean age of 37.6 years ranging from 5 to 73 years. In terms of age, $32 \%$ of the study sample were less than 30 years, while $28 \%$ of them were in their fifth decade. Male participants constituted $52 \%$ of the sample and $48 \%$ were female. The male to female ratio was 1.08:1. Most participants had sedentary jobs $(43 \%)$. Hematuria was associated with colic (58\%) and with equal sites of renal colic (24\%) for both right and left kidneys (Table 1).

Table 1: Age, sex, occupation, hematuria and site of renal colic of participants.

\begin{tabular}{|c|c|c|c|}
\hline Variables & Categories & No. & $\%$ \\
\hline \multirow[t]{2}{*}{ Gender } & Male & 52 & 52 \\
\hline & Female & 48 & 48 \\
\hline \multirow[t]{5}{*}{ Age(years) } & $\leq 30$ & 32 & 32 \\
\hline & $30-39$ & 24 & 24 \\
\hline & $40-49$ & 28 & 28 \\
\hline & $50-59$ & 6 & 6 \\
\hline & $\geq 60$ & 10 & 10 \\
\hline \multirow[t]{3}{*}{ Occupation } & Manual worker & 25 & 25 \\
\hline & Sedentary job & 43 & 43 \\
\hline & House worker & 32 & 32 \\
\hline \multirow[t]{3}{*}{ Hematuria } & Renal colic & 58 & 58 \\
\hline & Vague pain & 37 & 37 \\
\hline & Painless & 5 & 5 \\
\hline Total & & 100 & 100 \\
\hline
\end{tabular}


The role of ultrasound and intravenous urography .......

Zanco J. Med. Sci., Vol. 22, No. (2), August, 2018 https://doi.org/10.15218/zjms.2018.033

The findings of Table 2 reveal that 52 IVU diagnosed 17 as mild, nine as participants were diagnosed with renal stone by using ultrasound. In contrast, only 24 cases were diagnosed by IVU. The results of Table 3 indicate that among the different degrees of 27 hydronephrosis; moderate while one as severe hydronephrosis. In contrast, nine patients were diagnosed as mild, 17 as moderate and one as severe hydronephrosis by ultrasound.

Table 2: Diagnosis of renal stone by ultrasound and IVU.

\begin{tabular}{lcccc}
\hline Ultrasound & Yes & No & Total & $\begin{array}{c}P \text { value } \\
\text { (McNemar test) }\end{array}$ \\
\hline Yes & 24 & 28 & 52 & \\
No & $100 \%$ & $36.8 \%$ & $52 \%$ & $<0.001$ \\
& 0 & 48 & 48 & \\
Total & $0 \%$ & $63.2 \%$ & $48 \%$ & \\
& 24 & 76 & 100 & \\
\hline
\end{tabular}

Table 3: Comparison between ultrasound and IVU in detecting degrees of 27 hydronephrosis cases.

\section{IVU}

$\begin{array}{lccccc}\text { Ultrasound } & \text { Mild } & \text { Moderate } & \text { Severe } & \text { Total } & \begin{array}{c}P \text { value } \\ \text { (McNemar test) }\end{array} \\ \text { Mild } & 9 & 0 & 0 & 9 & \\ \text { Moderate } & 8 & 9 & 0 & 17 & 0.001 \\ \text { Severe } & 0 & 0 & 1 & 1 & \\ \text { Total } & 17 & 9 & 1 & 27 & \end{array}$


Table 4 shows that among the 100 patients, the ultrasound could detect 18 of them with ureteric stone which was more than the IVU of only 14 though the finding was not significant by the McNemar test. Ultrasound had higher ability $(79 \%)$ in detecting hydronephrosis than IVU (27\%). Both ultrasound and IVU had similar capabilities in diagnosing urinary bladder stones (6 cases for each) and renal masses (4 for each). McNemar test was not significant. The findings also reveal that ultrasound was superior in the diagnosis of bladder masses (10 cases) in comparison to IVU (3 patients only).

\section{Discussion}

Different imaging methods can be used for a patient with hematuria, each with its capabilities and disadvantages, hematuria either gross or microscopic, may be indicative of serious disease of the genitourinary tract, our study showed that hematuria is more frequent in men than in women. A systemic approach is required to choose diagnostic tools in hematuria cases. A comparison of ultrasound and IVU in our series was in favor of ultrasound for both urinary tract calculi and tumors. IVU lacks a high sensitivity in the diagnosis of renal tumors, particularly the small ones in the anterior or posterior lobe that have not impacted the anatomy of the collecting system. Also, if the patient is sensitive to contrast media or has a poor kidney function, IVU is contraindicated. On the other hand, given its low cost and noninvasive nature, ultrasound can be suggested as an alternative, regarding its accuracy in differentiating solid from cystic masses. ${ }^{16}$ Rafique and Javed studied the diagnostic accuracy of IVU and the

Table 4: Comparison between ultrasound and IVU in detection of different parameters.

\begin{tabular}{|c|c|c|c|c|}
\hline \multirow[b]{2}{*}{ US } & \multicolumn{2}{|c|}{ IVU } & \multirow[b]{2}{*}{ Total } & \multirow[b]{2}{*}{$\begin{array}{c}\text { P-value } \\
\text { (McNemar test) }\end{array}$} \\
\hline & Yes & No & & \\
\hline \multicolumn{5}{|c|}{ Ureteric stone } \\
\hline Yes & 14 & 4 & 18 & 0.133 \\
\hline No & 0 & 82 & 82 & \\
\hline Total & 14 & 86 & 100 & \\
\hline \multicolumn{5}{|c|}{ Hydronephrosis } \\
\hline Yes & 27 & 52 & 79 & \\
\hline No & 0 & 21 & 21 & $<0.001$ \\
\hline Total & 27 & 73 & 100 & \\
\hline \multicolumn{5}{|c|}{ Urinary bladder stones } \\
\hline Yes & 6 & 0 & 6 & \\
\hline No & 0 & 94 & 94 & 1.00 \\
\hline Total & 6 & 94 & 100 & \\
\hline \multicolumn{5}{|c|}{ Renal mass } \\
\hline Yes & 4 & 0 & 4 & \\
\hline No & 0 & 96 & 96 & 1.00 \\
\hline Total & 4 & 96 & 100 & \\
\hline \multicolumn{5}{|c|}{ Bladder mass } \\
\hline Yes & 3 & 7 & 10 & \\
\hline No & 0 & 90 & 90 & 0.023 \\
\hline Total & 3 & 97 & 100 & \\
\hline
\end{tabular}


transabdominal ultrasound in 100 patients with bladder carcinoma. They demonstrated that ultrasound is significantly more sensitive than IVU $(96 \%$ versus $87 \% ; P<0.01$ ). Also, ultrasound could determine the pathology of the upper urinary tract such as ureteral obstruction secondary to bladder cancer when IVU failed due to a poor kidney function. They suggested that ultrasound be used as a cost-effective method in cases of suspected bladder tumor. ${ }^{17}$ Moreover, Hoenig and coworkers have shown the value of ultrasound in 5 boys aged 11 to 18 years with transitional cell carcinoma. ${ }^{18}$ Ultrasound in the diagnosis of hematuria causes is able to show mucosal lesions greater than $4 \mathrm{~mm}$ to $5 \mathrm{~mm}$ when the bladder is full, while IVU could not show tumors smaller than $1.5 \mathrm{~cm}$. In 2005, Palmer and colleagues performed a study to determine the accuracy of ultrasound and CT scan without contrast in the diagnosis of urinary tract calculi in 75 children. Symptoms including flank pain and/or hematuria were present in $72 \%$ of the patients. They found that ultrasound could not detect the calculus in $41 \%$ of symptomatic patients, while CT scan was unable to show the calculus in $5 \% .{ }^{19}$ The Middleton and colleagues have shown a $91 \%$ sensitivity for ultrasound in the assessment of calculi remnants after percutaneous nephrolithotomy or shock wave lithotripsy. ${ }^{20}$ Marumo and coworkers have studied the hyperechoic spots accidentally found in the kidneys on ultrasound, They followed up 195 patients for 1 to 161 months and performed ultrasound on a yearly basis. Thirty-nine patients had hyperechoic spots while no calculi were detected on radiography. They underwent spiral CT scan with 3-mm cuts and calculi were seen in 31 (79.5\%). The authors reported that ultrasound is an effective diagnostic tool for finding calculi of patients with asymptomatic hematuria. ${ }^{21}$ Yilmaz and colleagues have studied 112 adult patients with renal colic, and a diagnosis of ureteral calculus was made by ultrasound, IVU, and CT scan. The sensitivity and specificity were $19 \%$ and $97 \%$ for ultrasound, $52 \%$ and $94 \%$ for IVU, and $94 \%$ and $97 \%$ for CT scan, respectively. ${ }^{22}$ Although Doppler ultrasound with the measurement of the resistive index and ureteral jet can increase the diagnostic value of ultrasound, ureteral calculi may not be detected when hydronephrosis and ureteral dilatation is not present, or when the patient is obese or has abdominal distention. We considered cases of pathologic hydronephrosis on ultrasound when a definite diagnosis was also calculi, as positive for ureteral calculi and also there were many cases of calculi proximate to the bladder. This can explain the high accuracy of ultrasound that we have found. IVU results were normal for hydronephrosis in these patients. Such cases warrant supplemental diagnostic measures. The results of ultrasound for lower ureteral calculi were superior to IVU; however, it is not a good diagnostic tool if hydronephrosis is absent and the calculus is not near to the ureterovesical junction. Complementary imaging may help us achieve a better result with ultrasound. For instance, using ultrasound and plain abdominal radiography as the first step. Henderson and colleagues reported a $97.1 \%$ sensitivity, higher than that of IVU, for urinary calculi in patients with hematuria and flank pain. ${ }^{23}$ They found that the likelihood of detecting a disease responsible for hematuria is higher when investigated by ultrasound compared with IVU (sensitivities, $85 \%$ versus $62.5 \%$ ). Mokulis and coworkers performed a study to assess patients with microscopic hematuria by ultrasound when the IVU results are normal; they found that $20 \%$ of 101 patients with a normal IVU result had abnormal findings on ultrasound. The authors concluded that ultrasound is necessary for patients with microscopic hematuria when IVU result is normal. ${ }^{24}$ A case-control study was done in Italy to compare the results of ultrasound in 516 
patients with hematuria and with those in 1788 controls. They reported a sensitivity of $93 \%$ and a specificity of $100 \%$ for the diagnosis of hematuria causes. ${ }^{25}$

\section{Conclusion}

Ultrasound is operator dependent, compared to IVU. However, many clinicians rely on ultrasound for the evaluation of patients with hematuria, especially when uremia, pregnancy, and other such conditions make IVU contraindicated. In the presence of less-invasive techniques such as shock wave lithotripsy, transurethral resection, transureteral lithotripsy, ureteroscopy, and cystoscopy, ultrasound findings may sometimes be stuffiest to make therapeutic decisions. However, we must decide to choose our diagnostic tool according to the patient's condition and the most suspected disorders causing hematuria. Raising awareness among physicians and urologist in our locality about the high accuracy of ultrasound in diagnosing hematuria and IVU should be preserved only for equivocal cases or when a detailed map of the pelvic-calyceal system is required.

\section{Competing interests}

The authors declare that they have no competing interests.

\section{References}

1. Alan J. Wein. Campbell-Walsh Urology. $9^{\text {th }}$ ed. Philadelphia, USA; 2007. P. 56.

2. Sutton D. Textbook of radiology and imaging, $7^{\text {th }}$ ed. Edinburgh: Churchill Livingstone; 2003. P. 970 .

3. Ryan S, McNicholas M , Eustace S. Anatomy for diagnostic imaging. $2^{\text {nd }}$ ed. Philadelphia: Saunders; 2004.

4. Drake RL, Vogl W, Mitchell AWM. Gray's Anatomy for Students. Philadelphia: Elsevier; 2005. P. 321 -3 .

5. Patel D, Patel U. Role of radiological imaging for the evaluation of hematuria. Trends in Urology and Men's Health 2011; 2(6):15-9.

6. O'Connor OJ, Fitzgerald E, Maher MM. Imaging of hematuria. Am J Roentgenol 2010; 195 (4):W263-7.

7. Ramchandani $\mathrm{P}$, Kisler $\mathrm{T}$, Francis IR. Expert Panel on Urologic Imaging. ACR Appropriateness Criteria hematuria. Reston, VA:
American College of Radiology; 2008.

8. Anderson EM, Murphy R, Rennie ATM, Cowan NC. Multidetector computed tomography urography (MDCTU) for diagnosing urothelial malignancy. Clin Radiol 2007; 62:324-32.

9. Handrigan MT, Thompson I, Foster M. Diagnostic procedures for the urogenital system. Emergency Medical Clinic. North America 2001; 19:745-61.

10. Miller OF, Rineer SK, Reichard SR, Buckley RG, Donovan MS, Graham IR, et al. Prospective comparison of unenhanced spiral computed tomography and intravenous urogram in the evaluation of acute flank pain. Urology 1998; 52:982-7.

11. Heneghan JP, Kim DH, Leder RA, DeLong D, Nelson RC. Compression CT urography: a comparison with IVU in the opacification of the collecting system and ureters. J Computed Assist Tomography 2001; 25:343-7.

12. Stoller M, Meng MV. Urinary stone disease. The practical guide to medical and surgical management $7^{\text {th }}$ ed. New Jersey: Hummana Press; 2002. P. 371.

13. Ansell G, Beltman MA, Complications in diagnostic imaging and interventional radiology. $3^{\text {rd }}$ ed. Oxford: Black Wall Science; 2004. P. 270.

14. Ionizing Radiation (medical exposure) regulations 2000, National Radiation Board, United Kingdom; 2000 (accessed August 1, 2016 at http//www.nr.pb.org.UK).

15. Dawson P, Sidhu PS. Is there a role for corticosteroid prophylaxsis in patients at increased risk of adverse reaction to intravenous contrast agent? Clin Radiol1993; 48(4):225-6.

16. Novick AC, Campbell SC. Renal tumors. In: Walsh PC, Retik AB, Vaughan ED Jr. Campbell's urology. $8^{\text {th }}$ ed. Philadelphia: WB Saunders; 2002. P. 2672-731.

17. Rafique $M$, Javed AA. Role of intravenous urography and transabdominal ultrasonography in the diagnosis of bladder carcinoma. Int Braz J Urol 2004; 30:185-90.

18. Hoenig DM, McRae S, Chen SC, Diamond DA, Rabinowitz R, Caldamone AA. Transitional cell carcinoma of the bladder in the pediatric patient. J Urol 1996;156:203-5.

19. Palmer JS, Donaher ER, O'Riordan MA, Dell KM. Diagnosis of pediatric urolithiasis: Role of ultrasound and computerized tomography. J Urol 2005; 174:1413-6.

20. Middleton WD, Dodds WJ, Lawson TL, Foley WD. Renal calculi: sensitivity for detection with US. Radiology 1988; 167:239-44.

21. Marumo K, Horiguchi $\mathrm{Y}$, Nakagawa K. Significance and diagnostic accuracy of renal calculi found by ultrasonography in patients with asymptomatic microscopic hematuria. Int J Urol 2002; 9:363-7.

22. Yilmaz S, Sindel T, Arslan G. Renal colic: comparison of spiral CT, US and IVU in the 
The role of ultrasound and intravenous urography .......

Zanco J. Med. Sci., Vol. 22, No. (2), August, 2018

https://doi.org/10.15218/zjms.2018.033

detection of ureteral calculi. Eur Radiol 1998; 8:212-7.

23. Henderson SO, Hoffner RJ, Aragona JL, Groth DE, Esekogwu VI, Chan D. Bedside emergency department ultrasonography plus radiography of the kidneys, ureters, and bladder vs intravenous pyelography in the evaluation of suspected ureteral colic. Acad Emerg Med 1998; 5:666-71.

24. Mokulis JA, Arndt WF, Downey JR, Caballero RL, Thompson IM. Should renal ultrasound be performed in the patient with microscopic hematuria and a normal excretory urogram? J Urol 1995; 154:1300-1.

25. Scialabba A, Li Vecchi M, Vigneri $S$. The ultrasonographic examination in hematuria. Evaluation of its diagnostic possibilities. Minerva Urol Nefrol 1992; 44:185-90. 